\title{
EL GRUPO DE WHATSAPP DE PADRES, ¿FACILITA O DIFICULTA LA EDUCACIÓN DE LOS HIJOS?
}

\author{
PARENTS' WHATSAPP CHAT GROUP. DOES IT MAKE CHILDREN'S \\ EDUCATION EASIER OR MORE DIFFICULT?
}

\begin{abstract}
$\overline{\text { Marta Chiquillo Enguix }{ }^{a} \text { y María José Llopis Bueno }}{ }^{b^{*}}$
\end{abstract}
Fechas de recepción y aceptación: 27 de septiembre de 2019; 14 de noviembre de 2019

Resumen: La modernidad fluida, tal como denomina Bauman al periodo en el que nos encontramos, se caracteriza principalmente por trascender los límites establecidos entre el espacio y el tiempo. El desarrollo vertiginoso de las tecnologías de la información y la comunicación ${ }^{1}$ y su uso generalizado ha afectado a todos los ámbitos de la sociedad. Entre ellos, destaca la revolución que ha provocado en las escuelas del siglo XXI, cuya dimensión social no puede entenderse sin las TIC; es decir, ha facilitado la comunicación de las familias con las escuelas, aumentando su participación debido a la facilidad que concede su uso a la generación millenials. Pero ¿todas las tecnologías han tenido efectos positivos? Algunas de ellas, como el uso del WhatsApp, han provocado posibles brechas en la relación familia-escuela, por lo que se propone una guía que ayude a las familias a hacer un uso correcto de esta aplicación que tantas posibilidades ofrece, con la finalidad de intentar levantar la barrera que esta aplicación ha provocado en la relación familia-escuela.

Palabras clave: modernidad fluida, tecnologías de la información y la comunicación, WhatsApp, millenials, relación familia-escuela, educación en valores.

${ }^{1}$ En adelante, TIC.

${ }^{a}$ Maestra en Primaria e Infantil en la Universidad Católica de Valencia San Vicente Mártir.

${ }^{\mathrm{b}}$ Departamento de Didáctica General, Teoría de la Educación e Innovación Educativa. Facultad de Magisterio y Ciencias de la Educación. Universidad Católica de Valencia San Vicente Mártir.

${ }^{*}$ Correspondencia: Universidad Católica de Valencia San Vicente Mártir. Facultad de Magisterio y Ciencias de la Educación. Calle Sagrado Corazón, 5. 46110 (Godella), Valencia. España.

E-mail: mariajose.1lopis@ucv.es. 
Abstract: Fluid modernity, as Bauman names the period in which we are living, is characterised by transcending the limits of space and the time. The rapid development of ICT and their generally use have affected all the areas in our society, including the revolution that it has caused in schools of the twenty first century, whose social dimension is not possible to understand without ICT; in other words, ICT has facilitated the communication between families and schools, increasing their participation by making it easier for the millennial generation to connect. However, have all the technologies had positive effects on education? Some of them, like WhatsApp, have caused potential gaps in the relationship between families and school, which is the reason why we proposed a guide to help families know how to use this app correctly, as much as it offers many possibilities when it comes to the communication between families and schools.

Keywords: fluid modernity, information and communication technologies, WhatsApp, millenials, family and school relationship, education in values.

\section{INTRODUCCIÓN}

Últimamente aparecen muchas noticias en los medios de comunicación sobre determinados problemas en las escuelas cuya causa se encuentra en el uso de la tecnología, especialmente de todos aquellos recursos vinculados al consumo de las redes sociales. Y estos problemas no se detectan exclusivamente en el alumnado escolar, sino que también aparecen en las relaciones que se establecen entre padres y maestros. El uso del móvil, acompañado de una de sus aplicaciones más utilizadas por toda la sociedad, WhatsApp, está suponiendo en muchos casos un grave problema en las relaciones familia-escuela.

Habitualmente se suele utilizar el cine para plasmar situaciones de la vida cotidiana y precisamente, en este sentido, se ha estrenado recientemente en los cines españoles un film del director y productor Santiago Segura (2019) que refleja la desbordada vida de un padre con sus cinco hijos y los diferentes grupos de WhatsApp a los que tiene que dar respuestas aprendiendo a discriminar lo verdaderamente importante de lo banal e insignificante. A partir de este ejemplo, podemos ser conscientes de que supone un problema enfrentarse a esta situación y aprender a lidiar con ella combinando las dificultades laborales y las necesidades de atención de cada uno de los hijos y cada una de sus circunstancias. Probablemente, muchos padres se sentirán identificados con esta situación. Pero ¿existe algún modo en el que el grupo deje de ser la 
pesadilla que es y se convierta en un nuevo método de comunicación fluida y provechosa entre la familia y la escuela?

Lo que pretendemos a través de este artículo es exponer las causas por las que se produce este hecho, que lo llega a convertir en una obsesión para muchas familias y, tras una reflexión debidamente fundamentada, dar una serie de pautas que faciliten y mejoren su uso en las relaciones entre la familia y la escuela.

\section{LA SOCIEDAD DE LA TECNOLOGÍA}

La sociedad, es decir, el conjunto de personas que conviven en un mismo espacio bajo un grupo de normas, ha ido cambiando a lo largo de los años, transformación que ha sido analizada por Bauman con mucho detenimiento, quien además acuña con el nombre de modernidad fluida un determinado periodo de nuestra historia reciente. Una de las grandes diferencias que señala este autor con respecto a épocas anteriores ha sido esa gran reducción en las diferencias existentes entre el espacio y el tiempo, y así nos encontramos, por ejemplo, que un mensaje enviado en un momento determinado, en pocos segundos ha recorrido kilómetros de distancia. Esta disminución del espacio con respecto al tiempo ha supuesto un desarrollo exponencial de los medios de comunicación, especialmente en la tecnología móvil. Thompson (1998) explica que se producen nuevos tipos de relaciones muy diferentes a la interacción cara a cara, acuñada con el nombre de interacción mediática. Castells (2007), por su parte, se refiere a una comunicación multimodal, es decir, a una transformación de la sociabilidad, debido a que el móvil permite una capacidad permanente de conectividad en cualquier espacio y momento, o como indica Turkle (2011) se crea una ilusión de compañía sin las exigencias que implica una amistad.

El mayor auge de la tecnología móvil se produjo a mediados de los años noventa, en concreto, en España, 96,8 de cada 100 habitantes utilizaban ya la telefonía móvil, y su uso se incrementó debido a un cambio en el sistema de tarifación de las llamadas, la interoperabilidad de las comunicaciones, de voz y datos, el incremento del número de minutos dedicados a hablar por el móvil, 
un descenso del precio del minuto de conversación, y un aumento en el tráfico de mensajes cortos. De entre los distintos sectores de la población fueron los más jóvenes los que con mayor fuerza la adoptaron (Castells, 2007).

En el año 2009, dos extrabajadores de Yahoo, Brian Acton y Jan Kuom, decidieron emprender un proyecto empresarial juntos, tras observar dos realidades que se estaban desarrollando exponencialmente en la sociedad: el surgimiento de un nuevo sistema operativo para teléfonos móviles y la expansión de las aplicaciones de iPhone. Este hecho supuso una gran oportunidad para la creación de una nueva aplicación, What's Up, que traducido al inglés significa "qué pasa", aplicación que poco a poco se ha ido convirtiendo en una de las más usadas por la sociedad debido, sobre todo, a su facilidad de conexión. Como el mercado de aplicaciones de iPhone no estaba muy sentado, los resultados no fueron los esperados. Sin embargo, que su servicio no llevase publicidad y no almacenara mensajes evitando poner en peligro la privacidad de los ciudadanos supuso que en cinco años WhatsApp se convirtiera en una de las aplicaciones más usadas. Debido a que cada vez había mayor cantidad de descargas de la aplicación, se desarrolló la versión Android y la posibilidad de enviar fotos, saltando años más tarde a la versión web (Rowan, 2014).

\subsection{Padres y madres nativos digitales}

La sociedad en la que vivimos actualmente difiere bastante de las que nos preceden, y ello conlleva consecuencias educativas considerables. Goethe (2012) expone los diferentes tipos de familias con los que nos podemos encontrar, identificando tres tipos con características diversas:

- Familias preocupadas por la educación de sus hijos. Están centradas en la educación de sus hijos, y por ello tratan de actualizarse y acudir a las escuelas de padres, así como a las tutorías que la profesora propone con la finalidad de facilitar que las cosas se desarrollen mejor cada día.

- Familias totalmente despreocupadas por la educación de sus hijos. Son familias que no se hacen cargo de educar a sus hijos, pensando que esta labor es totalmente de la escuela. No acuden a las actividades que 
la escuela propone y, además, no realizan un seguimiento de las tareas escolares. Asimismo, son padres que actúan como "abogados defensores" de su prole, ausentándose de la información que la escuela les transmite.

- Familias preocupadas en exceso por la educación de sus hijos. Este tipo de familias quiere mantener un contacto continuo con el profesor y tener a sus hijos completamente vigilados. Existe la posibilidad de que esta actitud de los padres tampoco sea completamente positiva para el alumno, puesto que en ocasiones puede suponer que el escolar se agobie en exceso por la constante vigilancia de sus padres.

La generación de millenials, también llamados nativos digitales, puesto que son capaces de aunar las TIC con la finalidad de sacar un mayor partido de ellas (De Haro, 2017), la conforman padres y madres que nacieron en la superabundancia y la rapidez, y se caracterizan por su uso constante de la mensajería instantánea, las redes sociales y el correo electrónico. Es decir, tienen integradas en sus vidas las TIC, y el uso que les dan a estas es intenso (Cuesta, 2017). Por tanto, podemos definir a los millenials como "la generación que ha crecido con internet como un gran referente informativo y que muestra su preferencia por los contenidos digitales" (Santín y Monzoncillo, 2017, p. 79).

La información de la que principalmente se nutre la generación millenial está centrada en las redes sociales, entre las que destaca Facebook, WhatsApp, Twitter, Instagram y Youtube, usados con el deseo de ampliar las amistades y acceder a la información actual. Los millenials son muy sociales, de forma que las redes sociales para ellos son una parte constitutiva en su modo de relacionarse con los demás. Y así, por ejemplo, Twitter la consideran una red para compartir noticias, opiniones y reflexiones, mientras que Instagram es la red elegida para compartir fotografías, hablar con amigos y conocer lo que hacen y dicen sus referentes; es por ello por lo que, para los millenials, las redes sociales son un nuevo espacio para hacer amistades y socializarse (Gutierrez Rubí, s. f.). 


\subsection{Educación coordinada: familia-escuela}

Argente y Llorca (2013) definen la comunicación familia escuela como el proceso en

el cual el maestro facilita a los padres una información sobre la actividad que realiza en el centro educativo y la familia aporta todo aquello que pueda afectar en el normal desarrollo del niño en la actividad escolar. Esta comunicación viene acompañada de una participación educativa, es decir, un compromiso por parte de las familias para desarrollar acciones que complementen la labor del profesorado.

En esta relación entre la familia y la escuela, estos autores señalan la existencia de unos niveles de participación que comportan una serie de actividades que los padres deben llevar a cabo.

FIGURA 1

Formas y niveles de participación

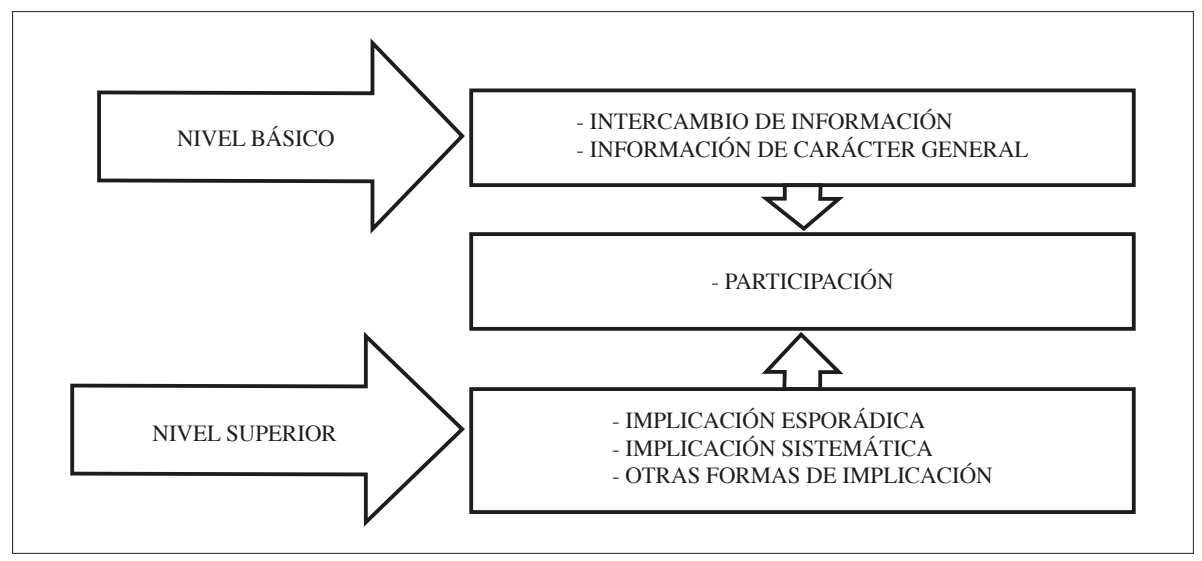

Fuente: elaboración propia a partir de Argente y Llorca (2013, p. 178)

Centrándonos en el nivel básico, es decir, el intercambio de información, como dicen los autores referenciados en la figura 1, a nivel individual, padres y maestros intercambian información de los niños que educan en común. 
El mejor método para conseguir acuerdos fructíferos y, por lo tanto, potenciar el sentimiento de pertenencia a un grupo es con el intercambio recíproco de datos y opiniones basado en el respeto, la responsabilidad compartida y la toma de decisiones conjunta (Macià, 2016, p. 74). Este método se centra principalmente en tutorías, y, por tanto, como señala Goethe (2012), los padres deben empezar a entender que las tutorías son de suma importancia, puesto que pretenden un compromiso y una mejora en la educación de los hijos. Así pues, podemos definir las tutorías como:

una reunión que mantenemos con el tutor de nuestro hijo para intercambiar información sobre él, sobre cómo va evolucionando y las estrategias educativas que vamos a adoptar de manera conjunta a lo largo del curso los padres y el profesorado para que se cumplan los objetivos educativos establecidos (González, 2016, p. 20).

Y con respecto al nivel superior señalado en la figura 1, debemos aclarar que supone dar un paso más; se trata de que los padres se impliquen en las actividades cotidianas de la escuela. Esta implicación puede ser, por un lado, esporádica, lo que según Argente y Llorca (2013) se produce cuando el maestro solicita una colaboración puntual de los padres con el centro educativo. Y, por otro lado, podemos referirnos a una implicación sistemática, pautada, coordinada y repetida en diversos momentos del curso escolar. Entre los agentes de implicación de los padres a nivel superior destacamos la AMPA, puesto que es el principal interlocutor entre las familias y el profesorado, y el Consejo Escolar, órgano que permite que toda la comunidad educativa pueda tomar decisiones de forma directa en el centro educativo (Goethe, 2012).

Existen tres razones que nos llevan a pensar que si se tiene en cuenta la participación en Educación Infantil podemos garantizar que se está estableciendo una garantía de calidad y de resultados eficaces en la escuela (Sáez y Subías, 2009). Las tres razones que nos llevan a ese pensamiento son: que los aprendizajes de los niños menores de seis años se encuentran enraizados a su vida cotidiana; que padres y profesores han de ser conscientes de la necesidad de estar complementados en la labor educativa de los hijos y que la implicación de los padres en el proyecto educativo de los hijos consigue un efecto positivo sobre el niño a largo plazo. 


\subsection{Comunicación con las familias en escuelas TIC}

La dimensión socializadora de la educación no puede entenderse actualmente sin la presencia de las TIC, a través de las plataformas digitales, redes sociales y el uso de dispositivos digitales, puesto que se posibilita que todos los miembros de la comunidad educativa interactúen y colaboren de forma participativa, repercutiendo lógicamente en la creación de dinámicas sociales inclusivas (Cano et al., 2014).

La introducción de las TIC ha abierto nuevas posibilidades de comunicación y nuevas perspectivas para informar e implicar a las familias. Ello se debe a que lo que se pretende es favorecer la conciencia de grupo y, por otro lado, abrir canales bidireccionales, con la finalidad del intercambio de información del grupo de clase (Macià, 2016).

Ciertamente, las TIC pueden ofrecer una forma alternativa de mejora para el proceso comunicativo en el ámbito de la acción tutorial, debido a que pueden contener letras, números y otros símbolos, caracterizados por la inmediatez, sencillez y rapidez de envío y recepción, abriendo canales de comunicación en cualquier espacio y en cualquier momento. La acción tutorial debe ser una responsabilidad compartida entre el profesor-tutor y su equipo educativo, basada en el seguimiento continuo del alumnado y procurando una intercomunicación continua y fluida. Las TIC pueden crear cauces de comunicación alternativos a los tradicionales que ayudarán a fomentar la participación de las familias en el proceso educativo del alumnado (Brazuelo, 2009, p. 54).

\section{El WhatsApp en las RElaciones familia-ESCUEla}

Como señalan Noa y Tali (2016), el teléfono móvil ha desarrollado aplicaciones de mensajería instantánea y, de entre todas ellas, cabe destacar WhatsApp, que se ha convertido en el servicio más utilizado a nivel mundial. Su rápida difusión viene dada por la comodidad de enviar mensajes de texto, imágenes y notas de voz rápidamente (Naidoo et al., 2018). Por estas razones, las interacciones vía WhatsApp son cada vez más frecuentes en todos los ámbitos institucionales. Y si nos centramos en la escuela, se producen interaccio- 
nes grupales, cuya activación de canal se da por supuesta; por ello, no existen cierres, lo que garantiza una continuidad en la conversación (Noblía, 2018).

Según datos extraídos de la Asociación Andaluza de Centros de Enseñanza de la Economía Social (ACES), especializada en protección de datos (2015), los grupos que se crean espontáneamente en los que solo participan padres están fuera de la Ley Orgánica de Protección de Datos (LOPD15/1999). Esta Ley, en su artículo 2.2.a, especifica que "el régimen de protección de datos de carácter personal que se establece en la presente ley no será de aplicación a los ficheros mantenidos por personas físicas en el ejercicio de actividades exclusivamente personales o domésticas". Sin embargo, en el caso de que el grupo sea creado por el propio centro, atendiendo a la política de privacidad de WhatsApp, en sus responsabilidades de seguridad, se establece que solo personas autorizadas puedan usar la cuenta, responsabilizándolas de todas las actividades que ocurran en la red social. Por ello, se establece que la empresa debe mantener la seguridad de credenciales de acceso a la cuenta, protegerla, evitar el acceso sin autorización y avisar de inmediato en el caso de sospechar cualquier vulneración de seguridad que la aplicación WhatsApp ofrece.

La Agencia Española de Protección de Datos (AEPD, 2018) tiene publicada una guía para centros educativos en la que se recoge que puede existir la excepción por la cual se contemple que sean los propios padres los que soliciten al tutor la creación de un grupo por circunstancias especiales como podría ser, por ejemplo, con alumnos con NEAE, pero es aconsejable en estos casos que se encargue de su gestión y creación un representante de las familias (un padre o una madre). Y con respecto a la difusión de imágenes e información de carácter personal con el grupo, señala que no se debe difundir como parte de la función educativa que ejerce el tutor. Sin embargo, si es un caso extremo, en donde el menor esté comprometido y con la única finalidad de tranquilizar al padre, se podrían captar imágenes y enviarlas, siempre con el consentimiento firmado de las familias.

\subsection{Tendencias actuales del uso de los grupos de WhatsApp en la escuela}

Cada día es más alta la frecuencia de uso de los grupos de WhatsApp en los centros educativos (Martínez, Cascales-Martínez y Gomáriz-Vicente, 2017). 
De entre los usos mayoritarios (Galatolo, 2018) destacan las apariencias que a través de los grupos de WhatsApp dan los padres. Se puede llegar a dar la sensación de que somos inferiores al resto de padres si no estamos atentos a todo. En muchas ocasiones, los padres hablan tanto por el grupo de WhatsApp que podrían llegar a olvidarse del trato cara a cara con sus propios hijos.

En el Informe del Defensor del Profesor 2017-2018 se avisa del aumento de denuncias por insultos y calumnias a los docentes a través de los grupos de WhatsApp, por lo que se puede afirmar que una de las tendencias actuales de su uso es el cotilleo y la difamación. Sin embargo, como señala González (2016), WhatsApp es una herramienta que permite estar en contacto con los padres y madres del centro, de forma que facilita la resolución de dudas, intercambio de información, reuniones, cumpleaños, trabajos de grupo. Aunque es cierto que, a pesar de estos usos tan beneficiosos, en muchos casos se está pasando a situaciones muy conflictivas. De entre ellas destacamos las críticas a la labor del profesor, la difusión de rumores que crean confusión, los intentos de solucionar los problemas con el profesor a través del grupo de WhatsApp $\mathrm{y}$, en último lugar, la creación de grupos alternativos, de forma que puedan compartir contenido sin que el resto de padres o el propio profesor sean conocedores de lo que se trama.

Por su parte, López-Cheda (2015) explica otro problema que ocasiona el uso del grupo de WhatsApp, que es la limitación de la autonomía de los niños, debido a que se trata de gestionar a través de estos grupos el tema de los deberes, consiguiendo que el niño no logre asumir sus responsabilidades, pues son sus adultos de referencia los que están pendientes de las tareas escolares.

\section{2 ¿Qué piensan las familias de los niños de Educación Infantil acerca de estos grupos?}

Con la finalidad de obtener datos contextualizados acerca de los usos dados por los padres de niños en Educación Infantil, durante el curso 2018-19 se ha pasado en un centro educativo de Valencia una encuesta elaborada por Inmaculada Martínez Hernández, Antonia Cascales-Martínez y María Ángeles Gomáriz-Vicente en el Departamento de Métodos de Investigación y Diagnóstico en Educación de la Universidad Murcia, en diciembre de 2017. 
Para su validación se seleccionó a un grupo de cinco expertos compuesto por docentes especialistas en educación y TIC. El nivel de fiabilidad del cuestionario fue analizado mediante la escala de Cronbach, y se obtuvo una puntuación global de 0,863 . Esta puntuación indica una alta consistencia interna.

La encuesta fue contestada por 90 padres y madres de niños en este ciclo. Nos sorprendimos al ver que los resultados coincidían con la elaborada en los centros públicos de Murcia en el año 2017. Hemos realizado un análisis de los resultados atendiendo a tres criterios: la normativa de uso, el fomento de la participación y la implicación en la escuela y el uso de esta aplicación como recurso educativo.

Con respecto a la normativa de uso, vemos cómo las familias afirman conocer muy bien el propósito de los grupos de WhatsApp, son conscientes de las normativas establecidas para divulgar y compartir información y muestran satisfacción por la manera en que se utiliza y gestiona el grupo de WhatsApp, tal y como se muestra en la tabla 1.

TABLA 1

Resultados de los items de la encuesta en la relación con el conocimiento por parte de los padres de la normativa de uso de los grupos de WhatsApp

\begin{tabular}{|l|c|c|}
\hline \multicolumn{1}{|c|}{ Ítems } & Si & No \\
\hline Conozco la finalidad del grupo de WhatsApp & $95,7 \%$ & $4,3 \%$ \\
\hline $\begin{array}{l}\text { Estoy satisfecho con la manera en que se utiliza y gestiona el grupo } \\
\text { de WhatsApp }\end{array}$ & $74,2 \%$ & $25,8 \%$ \\
\hline En el grupo de WhatsApp se cuestiona la figura del profesorado & $19,6 \%$ & $80,4 \%$ \\
\hline $\begin{array}{l}\text { Me ajusto a la normativa legal de lo que se puede publicar y compartir } \\
\text { en la escuela }\end{array}$ & $87 \%$ & $13 \%$ \\
\hline
\end{tabular}

Fuente: elaboración propia

Por otro lado, tal como comprobamos en la tabla 2, el uso de los grupos de WhatsApp no genera un gran compromiso e implicación en la educación de los hijos, puesto que, al no interferir desde el centro en el funcionamiento de los grupos, no repercute significativamente en el compromiso de los padres. Sin embargo, en su mayoría, los padres sí que hacen uso de otras herramientas del colegio, como la plataforma escolar. 
TABLA 2

Resultado de los items en relación con la opinión de las familias en cómo el grupo de WhatsApp fomenta la participación y la implicación en la escuela

\begin{tabular}{|l|c|c|}
\hline \multicolumn{1}{|c|}{ Ítem } & Si & No \\
\hline El grupo de WhatsApp favorece la implicación de los padres en la educación & $63,4 \%$ & $36,6 \%$ \\
\hline $\begin{array}{l}\text { Desde mi participación en el grupo siento que formo parte de un proyecto } \\
\text { educativo común }\end{array}$ & $45,2 \%$ & $54,8 \%$ \\
\hline $\begin{array}{l}\text { Gracias a la participación en los grupos de WhatsApp del centro me siento } \\
\text { parte activa y participativa de la educación de mis hijos }\end{array}$ & $55,9 \%$ & $44,1 \%$ \\
\hline Hago uso de la herramienta del colegio (Twitter, plataformas...) & $76,3 \%$ & $23,7 \%$ \\
\hline
\end{tabular}

Fuente: elaboración propia

Finalmente, comprobamos en la tabla 3 que las familias participantes consideran útil estos grupos en la medida en que les ayuda a organizar tareas escolares; sin embargo, no creen que sea una aplicación que optimice el rendimiento escolar de los hijos, ni que forje una mayor autonomía en sus estudios, aunque reconocen que ayuda a una mejor organización de las tareas escolares $\mathrm{y}$ en algunas ocasiones sirve de agenda escolar, debido a que se incentiva mucho por el grupo el envío de material didáctico.

TABLA 3

Resultado de los items en relación con la opinión de las familias del uso de los grupos de WhatsApp como recurso educativo

\begin{tabular}{|l|c|c|}
\hline \multicolumn{1}{|c|}{ Ítem } & $S i$ & No \\
\hline $\begin{array}{l}\text { Considero que pertenecer al grupo de WhatsApp facilita una mejor organiza- } \\
\text { ción de las tareas escolares }\end{array}$ & $76,3 \%$ & $23,7 \%$ \\
\hline $\begin{array}{l}\text { Pertenecer al grupo WhatsApp genera en mi hijo una mayor autonomía en sus } \\
\text { estudios. }\end{array}$ & $80,6 \%$ & $19,4 \%$ \\
\hline El WhatsApp es más útil que la agenda escolar & $67,7 \%$ & $32,3 \%$ \\
\hline Considero que el grupo de WhatsApp mejora el rendimiento escolar & $80,6 \%$ & $19,4 \%$ \\
\hline $\begin{array}{l}\text { Se incentiva por el grupo de WhatsApp el envío de material didáctico de } \\
\text { apoyo a la enseñanza. }\end{array}$ & $51,6 \%$ & $48,4 \%$ \\
\hline
\end{tabular}

Fuente: elaboración propia 
En conclusión, según la perspectiva de las familias, el centro escolar suele mantenerse al margen del uso de los grupos de WhatsApp de padres, puesto que no ofrecen información sobre su empleo ni regulan el tipo de publicaciones que se envían en él. Por ello, se destaca la importancia de establecer canales de comunicación de carácter bidireccional que ayuden a regular el manejo de este tipo de grupos.

Por otro lado, observamos que pese a la preocupación que existe con respecto a la legitimidad de la figura del profesor, los resultados no son tan significativos $\mathrm{y}$, en cambio, los padres sí que reconocen que lo utilizan para organizar a sus hijos las tareas escolares.

En conclusión, podríamos decir que el grupo de WhatsApp no es tan perjudicial como socialmente es considerado ni tan beneficioso como la generación millenials lo supone. Por ello, se considera necesario que las administraciones educativas sean conscientes de la utilización que se da a estos grupos con la finalidad de que promuevan sesiones informativas para concienciar a las familias sobre el buen uso del WhatsApp y sus consecuencias educativas, si está bien gestionado.

\subsection{Recomendaciones de buen uso de los grupos de Whats App de padres}

Tras todo lo expuesto, se presenta una serie de recomendaciones con la finalidad de que los grupos de WhatsApp dejen de suponer una situación problemática en las instituciones educativas y, en consecuencia, se conviertan en una nueva oportunidad de comunicación entre la familia y la escuela y en un nuevo método para facilitar la responsabilidad que comparten padres y maestros en la educación de los hijos.

1. Evitar el uso del grupo para criticar al docente: para la educación de los hijos se necesita una completa coordinación entre el maestro y la familia que facilite la educación de los hijos. Por ello, padres y maestros deben tener unos objetivos claros que lograr con respecto al niño, con la finalidad de procurarle un crecimiento y desarrollo equilibrado, así como la adquisición de habilidades y del conocimiento de valores que le conduzcan a convertirse en un buen ciudadano. Y en el mismo 
sentido, si un miembro del grupo decide criticar al maestro, hay que evitar ser cómplice de esas murmuraciones.

2. Selección de información: debemos limitarnos a compartir documentación e información referente a la escuela, evitando información de carácter personal. También se debe evitar la difusión de vídeos, fotos e imágenes del docente, madres, padres o alumnos, pues está penalizado por la Ley de Protección de Datos. Si la información va dirigida a un único individuo perteneciente al grupo, se debe tener la precaución de enviar ese contenido por privado a la persona interesada.

3. Permite a los niños ser responsables de sus tareas: es muy importante permitir a los niños tener autonomía en su trabajo y no acostumbrarse a que nadie haga las tareas por él. El WhatsApp muchas veces se convierte en la herramienta ideal para preguntar acerca de deberes, resultados de ejercicios o cómo llevar a cabo un trabajo, de manera que se fomenta el desinterés del alumno por anotar y estar atento a las indicaciones en el aula, ya que sabe que contará siempre con la intervención posterior de sus padres para solucionarle la gestión de estas tareas.

4. Los niños aprenden jugando: vivimos en sociedad, y convivimos con los demás siempre, de ahí radica la importancia de que los niños aprendan a socializar, tanto para su presente como para su futuro. La socialización se adquiere básicamente a través del juego. Algunas habilidades como comunicar, negociar, ceder o empatizar se aprenden jugando y, muchas veces, el abuso de la tecnología está privando a los menores de esa dedicación temporal a otro tipo de actividades lúdicas proactivas.

5. Utilizar el grupo de forma positiva: el WhatsApp permite una conexión y comunicación en tiempo real. Por tanto, si surge una duda de emergencia siempre es una vía de resolución de la situación. Y, además, puede ser utilizado para la comunicación de eventos y excursiones.

\subsection{Beneficios que se presentan con la irrupción de WhatsApp en los centros educativos}

El grupo de WhatsApp puede llegar a ser muy eficaz para informarse de lo que sucede en el centro escolar y para resolver dudas que puedan surgir entre 
las familias, incluso para llevar a cabo un intercambio de información sobre actividades escolares y extraescolares.

Según se recoge en Educación 3.0 (2018), el WhatsApp puede usarse como herramienta para la creación de escuelas de familias, puesto que en muchas ocasiones las escuelas de padres presenciales no acaban de tener éxito, por falta de tiempo y motivación. Por ello, ya que existen conferencias, charlas, artículos y vídeos de interés educativo, es muy viable la difusión de toda esta información a través de un grupo de WhatsApp de padres.

Las ventajas de ese servicio son, entre otras, la facilidad para que las familias inscritas reciban mensajes periódicos (dos veces a la semana, por ejemplo), la organización a través de una lista de difusión o el hecho de que estos mensajes incluyan consejos para la educación de los hijos, así como vídeos y enlaces web de diversos temas de interés.

\section{Conclusiones}

El análisis del cambio que se ha llevado a cabo en la sociedad, en la que todo es instantáneo y donde la inmediatez de transmisión de información es uno de los aspectos más relevantes de esta transformación, ha provocado un proceso de digitalización de la sociedad cuyo culmen se encuentra en el nacimiento, la rápida evolución y la difusión del WhatsApp. Una aplicación que nació en 2009 y que ha llegado ya, en muy poco tiempo, a todos los ámbitos de la sociedad.

Este aspecto, unido a las características que se han investigado en el uso de la tecnología en la generación que pertenece a la edad de los padres de los niños que en la actualidad cursan Educación Infantil, hace que el uso de esta aplicación sea elevado y la difusión de información a través de medios digitales muy frecuente.

Por tanto, a través de este trabajo, se ha pretendido analizar cómo se establecen las relaciones familia-escuela en la actualidad, con los avances más modernos. Estos avances han aportado facilidades a las familias para cumplir el objetivo que comparten con la escuela: la educación de sus hijos. Sin embargo, algunas de las aplicaciones más novedosas que han invadido nuestra 
sociedad, llegando a la escuela como un nuevo método para la comunicación, han supuesto una dificultad en esta relación.

En los centros educativos también ha entrado la tecnología, y no es novedad que el uso de WhatsApp esté cada día tan generalizado en este contexto como en tantos otros. Debido a este hecho, se concluye que desde las distintas partes implicadas se deban tomar una serie de precauciones. Por su parte, los centros deben evitar mantenerse al margen e informar a las familias de cómo deben usar el WhatsApp para que este deje de ser un obstáculo y se convierta en un medio, en una vía más abierta para las buenas relaciones entre la familia y la escuela, así como la educación conjunta de sus hijos. Las familias deberían comprender que el maestro nunca se va a posicionar en su contra, sino que entre padres y maestros se pretende buscar la mejor solución para permitir que los hijos crezcan sanos, felices y libres.

Por tanto, lo que se pretende en este artículo es levantar esa barrera y, a través de la reflexión, mostrar que esta aplicación de mensajería instantánea, que tanto facilita la comunicación a nivel mundial, puede convertirse también en un medio que facilite la transmisión de información entre la familia y la escuela, si se le sabe dar el uso correcto y adecuado.

Se considera que con esta propuesta se dejan muchas vías abiertas, ya que hacen falta variados estudios que analicen con detenimiento qué es lo que ocurre en estos grupos, pero especialmente creemos que se deben realizar desde diferentes puntos de vista; primero desde la perspectiva de los padres, también desde el criterio de los maestros y, sobre todo, desde la óptica legal, con la finalidad de poder controlar mejor aquello que se comunica por un grupo y cómo estos mensajes afectan a cada uno de sus miembros, así como a todos aquellos que no son integrantes, pero se convierten en objeto de la conversación.

Tras todo lo investigado, por tanto, se puede decir que la aplicación es óptima para la comunicación, dependiendo del uso que se haga de ella, y, por supuesto, atendiendo a las características del ámbito en el que se utilice. En la escuela, se debe usar con precaución y nunca convertirlo en un medio para la crítica y la burla. Establecer unas relaciones adecuadas entre la familia y la escuela debe ser objetivo prioritario para todo educador, y por ello nos vemos abocados a aprovechar las ventajas que nos aportan los nuevos sistemas de comunicación, tomando las debidas precauciones para realizar un uso ade- 
cuado y procurando la formación pertinente a los usuarios de cada uno de los sectores de la comunidad educativa: familias, profesionales de la educación y/o alumnos.

\section{BiBLIOGRAFÍA}

Agencia Española de Protección de Datos (marzo de 2018). Guía para centros educativos.

Argente, A. y Llorca, E. (2013). Las relaciones familia-escuela, en A. Argente, L. Ballester, E. Llorca, E. López, V. Marchante, J. M. Mula y M. Vilata, Docencia y práctica educativa. La estilística de la educación. Valencia: Boreal libros.

Asociación Andaluza de Centros De Enseñanza de Economía Social (2015). El WhatsApp y los centros educativos. Recuperado de: http:// boletin.acesandalucia.org/boletines/edicion165/6807_whatsapp $\% 20 \mathrm{y} \% 20$ centros\%20educativos.pdf .

BAUMAN, Z. (2000). La modernidad fluida. El Salvador (Argentina): Fondo de cultura económica de Argentina. Recuperado de: https://catedraepistemologia.files.wordpress.com/2009/05/modernidad-liquida.pdf

Brazuelo, F. G. y Galllego, J. D. (2009). Sistema de gestión tutorial vía SMS e internet. Revista de medios y educación 34, 49-67. Recuperado de: https://idus.us.es/xmlui/handle/11441/22591.

Cano, E. V., Meneses, E. L. y Zamora, L. C. (2014). La dimensión socializadora de la tecnología para una sociedad más abierta y colaborativa. Revista de la tecnología de Información y comunicación en educación 8(1), 145-155. Recuperado de: http:/www.riuc.bc.uc.edu.ve/bitstream/ 123456789/1118/1/art10.pdf .

Castells, M. (2007). Comunicación móvil y sociedad. Ed. Ariel / Fundación Telefónica.

Cuesta, M. (2017, diciembre). Radiografía social de los jóvenes adultos. Millenials. La generación emprendedora, 227-255. Recuperado de: https:// www.fundaciontelefonica.com/arte_cultura/publicaciones-listado/paginaitem-publicaciones/itempubli/588/. 
De Haro, G. (2017). El entorno competitivo: cambio, velocidad y tecnología. Millenials. La generación emprendedora, 41-71. Recuperado de: https:// www.fundaciontelefonica.com/arte_cultura/publicaciones-listado/paginaitem-publicaciones/itempubli/588/ .

Díez Gutierrez, E. (2011). Reseña de Comunicación y poder de Castells, Manuel. Revista Interuniversitaria de Formación del profesorado. Recuperado de: http://www.redalyc.org/articulo.oa?id=27422047013.

EduCACión 3.0 (2018). Cómo hacer un buen uso de WhatsApp entre familias y profesores. Recuperado de: https://www.educaciontrespuntocero.com/ experiencias/whatsapp-padres-alumnos-profesores/73269.html (consulta: 12/2018).

Educación, 3.0 (2018, 17 de mayo). Una escuela de familias vía WhatsApp. Recuperado de: https:/www.educaciontrespuntocero.com/experiencias/ whatsapp-escuela-familias/81480.html (consulta: 12/2018).

Goethe. J. W. (2012). La familia y la escuela ¿qué pueden hacer las familias por la educación? Madrid.

GonzÁlez, O. (2016) Familia y escuela, escuela y familia. Morata de Tajuña (Madrid): Descleé.

Gutiérrez-Rubí,A. (s. f.). Millenials en la era digital. Millenials en Uruguay. Valores, actitudes y comportamientos (pp. 16-26). Recuperado de: http://www. fundaciontelefonica.uy/wp-content/uploads/2018/12/MILLENNIALS _EN_URUGUAY-min.pdf .

LeY Orgánica 15/1999, de 13 de diciembre, de Protección de Datos de carácter personal. BOE-A-1999-23750. Recuperado de: https://www.boe.es/eli/ es/lo/1999/12/13/15.

MaciÀ, M. (2016). La comunicación familia-escuela: el uso de las TIC en los centros de primaria. Revista interuniversitaria de Formación del profesorado 19(1), 73-83. Recuperado de: http://dx.doi.org/10.6018/ reifop.19.1.2458.

Martinez, I. Cascales-Martínez, A. y Gomáriz-Vicente, M. (2017). Grupos de WhatsApp en familias de Educación Infantil y Primaria. Revista Latinoamericana de Tecnología educativa 16(2), 240-255. Recuperado de: https://relatec.unex.es/article/view/2940.

Naidoo, Stead, Mohangi, Zulu y Mbele (2018). Occupational therapy students use of social media for professional practice. Discipline of occu- 
pational therapy 10(2), 101-105. Recuperado de: https://www.ajol.info/ index.php/ajhpe/article/view/175664/165089 doi: 10.7196/AJHPE. 2018. v10i2.980.

NoblíA, V. (2018). La interacción laboral en redes sociales móviles. El uso de modos como estrategia de atenuación. Círculo de lingüística aplicada a la comunicación 73, 77-102. Recuperado de: http://dx.doi.org/10.5209/ CLAC.59060.

NoA, A. y TALI, G. (2016). The importance of the WhatsApp family group: an exploratory analysis. Aslib Journal of Information Management 68(2), $174-192$.

RowAn, D. (2014). WhatsApp: The inside story. Recuperado de: https://www. wired.co.uk/article/whatsapp-exclusive .

SÁez, L. y Subias, J. M. (2009): Educación Infantil. Ministerio de Educación, Gobierno de España: Aula mentor.

Santin, M. y Monzoncillo, J. A. (2017). El protagonismo de las redes sociales en la dieta informativa de los millenials. Millenials. La generación emprendedora, 79-101. Recuperado de: https:/www.fundaciontelefonica. com/arte_cultura/publicaciones-listado/pagina-item-publicaciones/itempubli $/ 588 /$.

Segura, S. (productor) y Segura, S. (director) (2019). Padre no hay más que uno (cinta cinematográfica). España: Atresmedia Cine.

Thompson, J. B. (1998). Los media y la modernidad. Una teoría de los medios de comunicación. Barcelona: Paidós. Recuperado de: https://cutt.ly/ Cw0MmPI.

TurkLE, S. (2011). Alone Together: Introduction, en S. Turkle, Alone toghether: why we expect more from technology and less from each other (pp. 1-23). Nueva York: Basic Books. Recuperado de: http://alonetogetherbook.com.

WhatsApp (2012). Información legal de WhatsApp. Recuperado de: https:// www.whatsapp.com/legal/. 
